\title{
How Practical Know-bow Contextualizes Theoretical Knowledge: Exporting Causal Knowledge From Laboratory to Nature
}

\author{
C. Kenneth Watersłf \\ Minnesota Center for Philosophy of Science, \\ Department of Philosophy, \\ University of Minnesota
}

\begin{abstract}
Leading philosophical accounts of classical genetics presume that Morgan's transmission theory can be understood independently of experimental practices. Experimentation is taken to be relevant to confirming, rather than interpreting, the transmission theory. But the construction of Morgan's theory went hand-inhand with the reconstruction of the chief experimental object, the model organism, Drosophila melanogaster. This raises an important question about theoretical knowledge gained in laboratory settings: when a theory (such as the theory of classical genetics) is constructed to account for phenomena in a carefully controlled laboratory setting, what knowledge, if any, indicates the theory's relevance or applicability to phenomena outside highly-controlled settings? The answer, I argue, is found within the procedural knowledge embedded within laboratory practice.

†Department of Philosophy, 831 Heller Hall, 271 19th Ave., University of Minnesota, Minneapolis, MN 55455-0310

$\ddagger \mathrm{I}$ would like to thank the audience at the 2006 Meeting of the Philosophy of Science Association for helpful comments.
\end{abstract}




\title{
How Practical Know-how Contextualizes Theoretical Knowledge:
}

\author{
Exporting Causal Knowledge From Laboratory to Nature
}

\section{Introduction.}

Thomas H. Morgan's theory of transmission genetics, like many scientific theories, was created in concert with the construction of an experimental system. Morgan's system was based on a species of fruit fly, Drosophila melanogaster. But experiments were not conducted on ordinary Drosophila flies. As Robert Kohler's history of Drosophila genetics shows, the fly was reconstructed to serve the purposes of Morgan and his colleagues (1994). Although Kohler focuses on what he calls "the moral economy" of the laboratory and ignores geneticists" theoretical work, his account suggests that the fly was reconstructed to exhibit inheritance patterns that could be explained with a relatively simple theory. And indeed, this was the case. The inheritance patterns explained by the transmission theory were not inheritance patterns exhibited by wild flies; rather, they were patterns generated by systematically changing the inner-composition of flies. The construction of the transmission theory of classical genetics went hand-in-hand with the reconstruction of the fly.

Using the transmission theory to account for the inheritance behavior of flies that were reconstructed so that their behavior would fit the theory is circular. But, it is not viciously circular with respect to explaining the inheritance behavior of the reconstructed flies. The theory truly accounted for inheritance patterns generated in the laboratory. But the fact that the theory accounted for these artificial patterns does not by itself imply that it also represented inheritance patterns in nature. And this raises an important epistemological question: what kind(s) of knowledge, if any, indicated the theory's relevance to inheritance in wild flies outside highly-controlled settings? The aim of this paper is to answer this question. ${ }^{1}$

\section{Received Model of Exportation of Laboratory Knowledge.}

One answer to our question about the exportation of knowledge from laboratory to nature might be called the "received model" because it relates to what has been called the received view of scientific theories. According to the latter view, scientific theories consist of universal, law-like statements and bridge

\footnotetext{
1 The issue I'm addressing is related to issues addressed in the scientific, philosophical, and historical literature on model organisms (see Ankeny 2001, Bolker 1995 and Bolker and Raff 1997, Fields and Johnston 2005, Rader 2004, and Schaffner 1998). But the literature on model organisms places more emphasis on the exportation of knowledge from a few species to many species whereas I am more interested in the exportation of knowledge from the laboratory to nature. My interests and ideas are more closely related to Cartwright's work in the philosophy of physics (1999 and Cartwright, Shomar, and Suarez 1995).
} 
principles. On this view, the exportation of theoretical knowledge from laboratory to nature is unproblematic because the knowledge gained in the laboratory is universal. The laws must apply to wild flies as well as reconstructed ones. This view does not deny the existence of practical difficulties involved with applying theories to concrete situations inside and outside the laborator The issue I'm addressing is related to issues addressed in the scientific, philosophical, and historical literature on model organisms (see Ankeny 2001, Bolker 1995 and Bolker and Raff 1997, Fields and Johnston 2005, Rader 2004, and Schaffner 1998). But the literature on model organisms places more emphasis on the exportation of knowledge from a few species to many species whereas I am more interested in the exportation of knowledge from the laboratory to nature. My interests and ideas are more closely related to Cartwright's work in the philosophy of physics (1999 and Cartwright, Shomar, and Suarez 1995). y. Such applications entail filling in the initial conditions, and the complications exhibited in nature may pose severe practical limitations. Hence, in practice, it might be difficult to apply a theory to individual cases in nature. Nevertheless, in principle, if a theory truly accounts for the laboratory phenomenon by identifying the underlying universal laws responsible for the laboratory phenomena, then it must represent the phenomena in nature as well.

According to this traditional model of exportation, scientists reconstruct flies in ways that reveal universal laws about the inheritance behavior of flies, which because they are universal, apply to wild flies as well. The difficulties in exportation are merely pragmatic. The received model might be summarized as follows:

study of artificial cases $\Rightarrow$ universal laws $\Rightarrow$ universal exportation

The problem with this model is that it depends on the idea that experimentation uncovers universal laws, but the principles that Morgan and his colleagues identified were not universal, at least not in the sense necessary for the received model of exportation.

\section{The Central Principles of the Transmission Theory.}

Classical geneticists, starting with Morgan and his collaborators, explained the transmission of phenotypic differences by following the transmission of gene differences from generation to generation and attributing the presence of alternative phenotypic traits to the presence of alternative forms of genes. Their theoretical explanations depended on the idea that genes are located in linear fashion on chromosomes, on principles about the transmission of genes that were grounded in an understanding of cytological processes such as meiosis, and on the principle that differences in genes cause differences in phenotypes. I will illustrate the problem with the received model by examining two of its key principles: the crossover principle and the difference principle. 
Crossing over occurs during meiosis, the process in which a diploid cell divides to produce haploid gametes. During this process, homologous chromosomes are aligned with one another and pulled apart. When they are aligned, they sometimes break and reconnect to the corresponding part of the homologous chromosome. This is called crossing over. Two genes located on a single chromosome, say the maternally derived chromosome, can be separated, if a single crossover event occurs between them. For instance, consider the recombination two genes located on the same chromosome, each of which has two different allelic forms. Call the allelic forms of the first gene, $A$ and $a$, and of the second gene, $B$ and $b$. Suppose a maternally derived chromosome contains an $A$ allele at one locus and a $B$ allele at another locus (is " $A B$ "). Suppose the paternally derived homologous chromosome contains $a$ at the first locus and $b$ at the second (is " $a b$ "). A single crossover event between these loci would result in $A b$ and $a B$ chromosomes. What I call the crossover principle can be stated as follows:

crossover events between genes on homologous chromosomes occur regularly and at rates is proportional to the distance separating the genes

This principle applies only to female Drosophila because crossing over does not occur in male flies. The regularity of crossover rates led to regularities in the recombination of genes during sexual reproduction, which resulted in observed regularities in the recombination of traits that appear together in the individual parents. Applying the crossover principle enabled geneticists to explain statistical patterns of inheritance and to determine where genes were located on chromosomes. This principle was absolutely critical to the practice of classical genetics.

The difference principle was just as important. It is what enabled geneticists to draw a connection between the genetic make-up of an individual and its outward phenotypic appearance. It associated the difference between two alternative alleles of a gene with alternative phenotypes:

differences in a gene cause uniform phenotypic differences in particular genetic and environmental contexts

Classical geneticists explained the transmission of characteristics from one generation to another in two stages. First they explained the transmission of genes from parents to offspring by drawing upon information about the location of genes and cytological principles such as the crossover principle. In the second stage, they drew upon information gained in the first stage about the genetic make-up of progeny and upon instantiations of the difference principle to explain the appearances of offspring.

The literature in philosophy of biology articulates a number of reasons for doubting that principles such as the crossover principle and difference principle represent universal laws (e.g. such as Beatty 1981 and Kitcher 1984). I won't repeat them here. It is time to move on, to develop an alternative interpretation of 
these principles, an interpretation that does not presuppose the ideal of universality. To do so, I will draw upon James Woodward's manipulability account of causal generalizations in science (2003).

\section{Woodward's Manipulability Account of Causal Generalizations.}

According to manipulability theories, causes can be understood as "handles" for bringing about effects in the sense that causes can be manipulated to bring about different outcomes. By manipulating a causal property, one can change an effect property. Woodward (2003) conceives of causal relationships as relationships between variables, so he would express this idea by saying that the value of the effect variable, $Y$, can be changed by manipulating the value of a causal variable, $X$. Roughly speaking, Woodward says a causal relationship exists between two variables, $X$ and $Y$, when:

for at least some individuals there is a possible manipulation of some value of $\mathrm{X}$ that they possess which, given other appropriate conditions (perhaps including manipulations that fix other variables distinct from $\mathrm{X}$ at certain values), will change the value of $\mathrm{Y}$ or the probability distribution of $\mathrm{Y}$ for those individuals. (Woodward 2003, 40)

Woodward's theory, as the inclusion of the causal term 'change' in the above passage indicates, is not reductive. It does not account for cause is in non-causal terms. It is a non-reductive, counterfactual theory, potentially consistent with different reductive theories, including counterfactual theories such as the one proposed by David Lewis (1973).

On Woodward's account, causal relationships are not necessarily universal. $X$ can cause $Y$ even if intervening on the value of $X$ within many ranges of the variables $X$ and $Y$ would not change the value of $Y$. For example, there is a causal relationship between temperature and the phase state of water even though increasing the temperature of water from $20^{\circ} \mathrm{C}$ to $95^{\circ} \mathrm{C}$ will not change its phase state. In addition, $X$ can cause $Y$ even if intervening on the value of $X$ has no effect on the value of $Y$ when the value of certain variables not identified in the expression ' $X$ causes $Y$ ' is outside some range. For example, the claim that raising the temperature of water from $20^{\circ} \mathrm{C}$ to $100^{\circ} \mathrm{C}$ causes water to boil is not contradicted by the fact that increasing the temperature of water from $20^{\circ} \mathrm{C}$ to $100^{\circ} \mathrm{C}$ will not change the phase state of water when the atmospheric pressure is significantly greater than standard atmospheric pressure.

Woodward says that scientists identify genuine causal relationships even though these relationships (for example, the ones specified in textbooks) are almost always "sensitive" to the values of variables that figure into the specified relationships and/or to the values of variables that do not figure into the specified relationships. According to his account, the expression ' $X$ causes $Y$ ' just means there are at least two different values of $X, \mathrm{x}_{1}$ and $\mathrm{x}_{2}$, and at least one set of values 
for the variables specified and the variables not specified in the expression, such that if one intervened to change the value of variable $X$ from $\mathrm{x}_{1}$ to $\mathrm{x}_{2}$, the value of $Y$ would change. The invariance of a causal relationship concerns ranges of values under which the relationship holds. Since causal relationships can be sensitive to the values of many different variables, and since the sensitivity with respect to one variable can depend on the value of other variables, the relevant 'ranges of values' refers to a space (or set of spaces) in a $\mathrm{n}$-dimensional array of variables (where $\mathrm{n}$ is the number of variables to which ' $X$ causes $Y$ ' is sensitive). Hence, it is more accurate to speak of "spaces of invariance" rather than a "range of invariance."

An advantage of Woodward's theory is that it accounts for the intuitively plausible idea that scientists typically explain phenomena by identifying causal dependencies that do not obtain under all conditions. Explaining why a flask of water boiled over the flame of a Bunsen burner does not depend on identifying a totally invariant generalization. It suffices to identify the change in water temperature as the cause of the change in phase state under the laboratory conditions.

\section{A Causal Interpretation of the Difference Principle.}

Woodward's theory provides a natural framework for expressing the causal relationships identified by classical geneticists. I will illustrate this point by using his framework to interpret the difference principle:

differences in a gene cause uniform phenotypic differences in particular genetic and environmental contexts

for at least some organisms, a possible manipulation of the form of a gene they possessed as a zygote, given other appropriate conditions (such as genetic background and environmental conditions), would change the phenotypes of those individuals

This reformulation makes salient features of the causal relationship expressed by the difference principle that are crucial for the practice of classical genetics. Experimentation in this science entailed controlling the genetic background and environmental conditions. Drosophila geneticists bred flies to "clean up" their genetic backgrounds so phenotypic effects of particular mutations would not be masked. They also controlled environmental conditions in the laboratory. These efforts resulted in regular and repeatable patterns of inheritance. Woodward's theory emphasizes the fact that causal relationships, such as those expressed by the difference principle, are not universal.

According to this interpretation, instantiations of the difference principle pertaining to the claim that a particular difference in genotype caused particular differences in phenotype could be used to explain transmission patterns generated 
in the laboratory even though these relationships were highly sensitive (a fuller account of the causality involved here can be found in Waters, forthcoming). Furthermore, it shows how the explanations did not depend on specifying the invariance space of the causal relationships. Provided a causal relationship was realized in the experiment set-up, the relationship could be used to explain the actual results. The fact that the relationship would not have been realized had the genetic background been different would be irrelevant to explaining the patterns generated when the background was carefully controlled. Hence, specifying the possible, but not actualized, range of genetic backgrounds under which the relationship would failed to hold is not necessary.

This interpretation, however, implies that the received model of exportation does not apply to laboratory genetics because the study of flies, reconstructed so causal principles like the difference principle would apply, yielded causal principles that were invariant with respect to the reconstructed flies under laboratory conditions, but not necessarily invariant over the range of conditions found in wild flies in nature. The theoretical explanations of what happened in the laboratory did not contain information about the invariance spaces of the causal generalizations invoked in the laboratory. ${ }^{2}$ So, the questions is, what knowledge, if any, provided information about whether the causal relationships extended to flies in nature. The information, I will suggest, was in the procedural knowledge.

\section{Procedural Knowledge.}

When classical geneticists explained inheritance patterns, they contributed to and drew upon pools of knowledge (for a fuller account see Waters 2004). Some of this knowledge was theoretical in the sense that it involved identifying underlying causal processes and constructing abstract models or representation structures. But much of the knowledge was practical in nature. In this section, I will describe the pool of procedural knowledge. In the following section, I will explain why the transmission theory cannot be properly understood unless it is placed in the context of the larger body of knowledge that includes the practical knowledge discussed in this section.

The procedural knowledge of classical genetics took different forms. Much of it was unwritten and was therefore passed on directly from experimenter to experimenter. Many researchers visited the Morgan laboratory to gain practical knowledge of Drosophila genetics. But a good share of the practical knowledge was communicated in written form. Early technical reports published by the Carnegie Institution of Washington and the Drosophila Information Service were filled with practical information. ${ }^{3}$ On the basis of written sources, one can distinguish

\footnotetext{
2 This resonates with a them in the literature on the semantic view of theories which distinguishes between theoretical models and hypotheses about how the models fit various parts of the world (e.g. see Giere 1988).

3 Carnegie Institution of Washington publications numbers 237 (1916), 278 (1919), 327 (1923), and 421 (1931). Reports of the Drosophila Information Service were edited by Bridges and
} 
three broad categories of practical knowledge in classical genetics. Undoubtedly, much additional detail could be added to this preliminary account.

\subsection{Special Methods of Experimentation.}

One category of practical knowledge involved special methods of experimentation. For example, geneticists collected knowledge about methods for identifying particular genotypes. Genetic analysis included studying the course of an allele's phenotypic expression throughout development. Knowledge from such studies helped researchers determine the stage at which a particular phenotype could be identified most easily and the trait(s) that should be used for identification. But geneticists also knew special tricks for identifying obscure mutants. One of these was called 'intensification' (or 'enhancement). This was first tried on purple mutants when researchers noticed that the expression of purple was intensified in flies homozygous for vermilion. That is, the difference between the purple and not-purple individuals is greater in a stock homozygous for vermilion than in a stock of wild-type flies. The early experiments on purple were carried out among individuals homozygous for vermilion to facilitate the separation of purples from not-purples. This practice was suspended as researchers developed confidence in their ability to separate purples from notpurples in wild-type stocks. But it continued to be important in the study of other genes (and continues to be used today). Intensification enabled geneticists to experiment on mutants that would otherwise have been too obscure for careful genetic analysis.

It is tempting to think that this category of practical knowledge had little connection to how geneticists understood heredity. We might conclude that it was important as a tool for gaining knowledge about heredity, but did not actually impinge on that understanding. But this kind of practical knowledge contained information about developmental genotypes that is obscured when one looks at the explanations of transmission patterns, which treated phenotypes as if they appeared all at once. The theoretical explanations didn't contain information about developmental dimensions of phenotypic expression. The information was embedded within the procedural knowledge. The next example illustrates practical knowledge that undoubtedly influenced the way practicing geneticists interpreted theoretical formulations of classical genetics.

Classical textbooks of genetics sometimes discussed mapping procedures and genetic distances as if the crossover rates between any two genes was constant (e.g. Morgan 1926, Sturtevant and Beadle 1939). In fact, crossover rates were not absolute. They varied from experiment to experiment. Through careful

Demerec and every issue contained articles on practical methods. The demand for such information was so great that the 6th number was published as a special issue completely devoted to methods. Bridges and Brehme 1944 also provided important practical information about methods. Methods sections of research articles often presuppose working knowledge of the methods described. The description of methods in the publications cited here, however, are far more informative. 
study, geneticists gained knowledge about how to obtain constant crossover rates. Tricks included maintaining the right temperature, testing for crossover in females alone, breeding females of the same age, and cleaning stocks of crossover modifiers. Implementing such techniques enabled geneticists to collect reproducible measurements of crossover rates. But these measurements, and the genetic maps that were based upon them, did not measure absolute rates of crossing over between loci or rates that would exist in wild flies in nature. What they measured was the relative rate of crossing over in artificially reconstructed flies under artificial laboratory conditions. The knowledge embedded within the methods and tricks of classical genetics had important implications for understanding how the theoretical explanations of laboratory phenomena might apply outside the laboratory.

\subsection{Empirical Information of Practical Importance.}

Implementing the general investigative strategies as well as the more specialized methods drew upon and contributed to a pool of detailed information. ${ }^{4}$ An example was the empirical generalization that crossing over does not occur in male Drosophila. This piece of information was crucial for designing the most common investigative strategies (e.g. the backcross test) as well as for devising protocols for material production (see section 3.3). This fact becomes absolutely clear in Bridges and Morgan's account of the practical difficulties of employing the backcross test before it was known that crossing over did not occur in male Drosophila (1919, pp. 173-4). Yet, because this fact is crucial for its practical rather than theoretical import, it is not the kind of detail that would be mentioned in a traditional philosophical accounts of scientific knowledge. Classical genetics was teeming with information of practical importance. Some of it was general such as the lack of crossing over in male Drosophila. Some was specific such as the observation that the development of certain mutants require colder or warmer developmental conditions (Bridges and Brehme 1944, see pp. 5 6). Such information was constantly being collected and stored, to be drawn upon when useful for furthering investigation or understanding. This pool of information provided geneticists with the context for interpreting the generality of their transmission theory. And because this information was vital for the everyday practice of genetics, it must have been especially conspicuous to practicing geneticists.

\subsection{Methods of Material Production and Maintenance.}

The knowledge of classical genetics included practical information about how to carry out procedures designed for producing and maintaining physical or biological products to be utilized in subsequent experiments. This knowledge was essential for practicing genetics. Some of this know-how admittedly involved

\footnotetext{
${ }^{4}$ See Waters 2004 for an account of the investigative strategies of classical genetics.
} 
matters far removed from reasoning about heredity. It is hard to get excited about recipes for the production of media suitable for sustaining mutant Drosophila in the laboratory, but knowledge of such mundane matters was nevertheless important to the practice of genetics (Kohler 1994). Improved media enhanced the viability of marginal mutants and hence expanded the range of mutants that could be genetically analyzed. It also simplified experimental methods by eliminating the necessity of carrying out certain balancing trials. The development of suitable media was essential for establishing Drosophila as a super model of experimental organisms.

Not all knowledge of material production was peripheral. Much of this knowledge was closely connected to the way classical geneticists understood heredity. Even the simple method for 'cleaning' new mutant stocks, which involved repeatedly crossing the mutant to wild-type and selecting for the character of interest, was thought of in terms of the classical transmission theory. More closely associated with the special knowledge of genetics were the procedures for obtaining stocks necessary for the kinds of investigative experiments described in section 2. The three-point backcross test, for instance, required obtaining a stock of triply homozygous recessives. This was an extremely challenging task because of the lack of crossing over in male Drosophila. Standard protocols for obtaining multiple homozygous recessives, which entailed cleverly arranged breeding and selection regimens, formed an integral part of the knowledge of classical genetics.

The practical knowledge of classical genetics included clever tricks for maintaining stocks as well. For example, Muller (1918) devised a strategy for preserving heterozygosity in stocks. This was especially useful for retaining recessive lethal mutations that would disappear in regularly kept stocks because of selective pressure. Muller's trick was to balance two lethal mutants, at neighboring loci, against one another by breeding individuals with one copy of each recessive on different members of a pair of homologous chromosomes. The presence of a recessive lethal on each chromosome forces heterozygosity because only the heterozygotes survive. Hence, there would be no selection against the lethal recessive alleles. Crossovers in females could defeat the system, so Muller added a crossover repressor. Designing and carrying out breeding regimens for producing and maintaining stocks could not have been achieved in the absence of explanatory reasoning. But, the focus of the knowledge here was on practical problems of material production and maintenance, not on explanatory problems of accounting for the transmission of phenotypic traits.

The procedural knowledge described in this section were largely aimed towards making experiments work. That is, they were aimed towards constructing a system that fell within the invariance spaces of the causal principles of the transmission theory. Geneticists knew that the crossover principle did not apply generally, so they reconstructed the fly, for example by breeding out crossover reducers, to produce organisms that obeyed the crossover principle. In effect, they were resetting the value of background variables so the causal relationship designated by the crossover principle would hold. This would be a problem if 
they were seeking universality in the crossover principle, but they weren't. Their real aim was to investigate biological processes by manipulating them. And to do this, they needed to make the experiments work. My thesis is that their knowledge about what it took to make the experiments work provided an important source of information about the extent to which principles of the transmission theory might apply outside the laboratory.

\section{Exportation of Causal Knowledge From the Laboratory.}

According to the received model of exportation, scientists construct artificial situations in the laboratory in order to reveal universal laws that apply everywhere, not just within the confines of the laboratory.

study of artificial situations $\Rightarrow$ universal laws $\Rightarrow$ universal exportation

The model of exportation I wish to propose differs in important ways. First of all, according to the account advanced here, laboratory research involves a process of dual construction. The fly wasn't simply reconstructed to remove disturbing elements that obscured universal principles. Rather, the fly was continually being reconstructed to bring the experimental system within the invariance spaces of the causal principles of the transmission theory. The difference principle didn't automatically apply to newly discovered mutants. Geneticists had to change genetic backgrounds so the difference principle would apply. Hence, a more accurate picture of the relationship between the study of artificial situations and the theoretical knowledge of genetics would convey an interplay between the construction of artificial situations and the formulation of somewhat invariant causal generalizations:
construction of
formulation of somewhat
laboratory system
$\Leftrightarrow$
invariant causal principles

The exportation of knowledge about the somewhat invariant causal principles, which are exemplified in carefully constructed laboratory systems, to the world outside the laboratory is informed by an understanding of the interplay between the construction of laboratory system and the formulation of the causal principles:

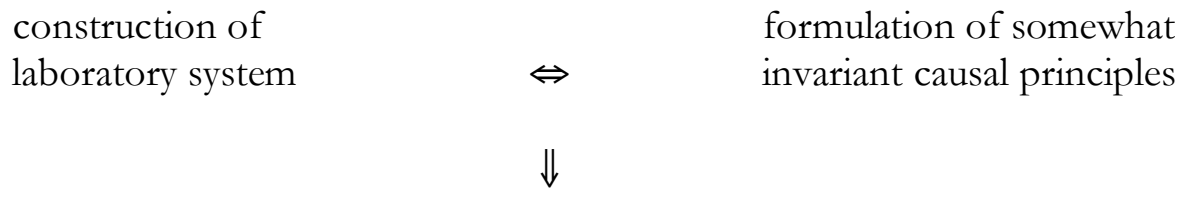

exportation of knowledge outside the laboratory 


\section{Conclusion.}

The explanatory and investigative practices of classical genetics were interwoven. Neither can be properly understood separately from the other. Explanatory reasoning depended on practical knowledge because the explanations appealed to causal regularities that were understood in light of knowledge about the procedures used to observe them. Without this practical knowledge, the scope of the regularities, even as applied to a single species, would have been unknown. Perhaps the clearest illustration of this point involves the crossover principle. Explanations of the recombination of phenotypic traits cited regular rates of crossing over (between given loci). But geneticists knew that crossover rates were variable. Rates were constant only when stocks were cleaned of crossover modifiers, bred at early ages, and maintained under appropriate laboratory conditions. In the absence of this practical knowledge, one might think that the regularities of crossing over apply to Drosophila generally when in fact they apply only to artificially modified flies under special conditions. This practical knowledge does not undermine the individual explanations of classical genetics or the causal regularities on which they depend. But it provides a more realistic understanding of them, an understanding more in keeping with the knowledge of practicing geneticists. 


\section{References}

Ankeny, Rachel A. (2001), "Model Organisms as Models: Understanding the "Lingua Franca' of the Human Genome Project", Philosophy of Science, 68: S251-S261.

Beatty, John (1981), "What's Wrong with the Received View of Evolutionary Theory", in P. D. Asquith and R. N. Giere (eds.), Vol. 2 of PSA 1980, East Lansing, MI: Philosophy of Science Association.

Bolker, Jessica A. (1995), "Model Systems in Developmental Biology", BioEssays 17, No. 5: $451-455$.

Bolker, Jessica A. and Raff, Rudolf A. (1997), "Beyond Worms, Flies, And Mice: It's Time To Widen The Scope Of Developmental Biology", The Journal of NIH Research, 9: 35-39

Bridges, C. B. and Katherine S. Brehme. (1944), The Mutants of Drosophila melanogaster. Washington, DC: Publications of the Carnegie Institution of Washington.

Bridges, Calvin B., and Thomas H. Morgan (1919), "The second-chromosome group of mutant characters", in Contributions to the Genetics of Drosophila Melanogaster, Washington, DC: Carnegie Institution of Washington publication no. 278: 123-304.

Cartwright, Nancy (1999), The Dappled World, Cambridge: Cambridge University Press.

Cartwright, Nancy, Towfic Shomar, and Mauricio Suarez (1995), "The Tool Box of Science: Tools for the Building of Models with a Superconductivity Example", Poznan Studies in the Philosophy of the Sciences and the Humanities, 44:137-149.

Fields, Stanley and Johnston, Mark (2005), "Whither Model Organism Research?”, Science 307, Issue 5717: 1885-1886.

Giere, Ronald N. (1988), Explaining Science, Chicago, IL: The University of Chicago Press.

Hacking, I. (1983), Representing and Intervening, Cambridge: Cambridge University Press.

Kitcher, Philip S. (1984). "1953 and All That. A Tale of Two Sciences", The Philosophical Review, 43: 335-371.

Kohler, Robert (1994), Lords_of the Fly: Drosophila Genetics and the Experimental Life. Chicago: Chicago University Press.

Lewis, David (1973), "Causation", Journal of Philosophy, 70: 556-67.

Morgan, Thomas H. (1926), The Theory of the Gene, New Haven, CT: Yale University Press.

Muller, Hermann J. (1918), "Genetic variability, twin hybrids and constant hybrids, in a case of balanced lethal factors", Genetics 3: 422-99.

Rader, Karen A. (2004), Making Mice: Standardizing Animals for American Biomedical Research, 1900-1955, Princeton, NJ: Princeton University Press.

Schaffner, Kenneth F. (1988), "Model Organisms and Behavioral Genetics: A Rejoinder", Philosophy of Science, 65: 276-288.

Sturtevant, Alfred H., and George W. Beadle (1939), An Introduction to Genetics, Philadelphia, PA: W. B. Saunders Company.

Rheinberger, Hans-Joerg (1997), Towards a History of Epistemic Things, Synthesizing Proteins in the Test Tube. Stanford. CA: Stanford University Press

Waters, C. Kenneth (2004), "What was Classical Genetics?", Studies in History and Philosophy of Science 35: 783-809"

Waters, C. Kenneth (forthcoming), "Causes that Make a Difference", The Journal of Philosophy

Weber, Marcel (2005), "Model Organisms: Of Flies and Elephants", in Philosophy of Experimental Biology, Cambridge University Press: Cambridge (2005): 154-187.

Woodward, James (2003), Making Things Happen Oxford: Oxford University Press. 